\title{
Perimembrane Aurora-A Expression is a Significant Prognostic Factor in Correlation with Proliferative Activity in Non-Small-Cell Lung Cancer (NSCLC)
}

\author{
Eiji Ogawa, MD, ${ }^{1,2}$ Kazumasa Takenaka, MD, PhD, ${ }^{1}$ Hiromichi Katakura, MD, PhD, ${ }^{1}$ \\ Masashi Adachi, MD, ${ }^{1}$ Yosuke Otake, MD, PhD, ${ }^{1}$ Yoshinobu Toda, PhD, ${ }^{3}$ \\ Hirokazu Kotani, MD, PhD, ${ }^{2,3}$ Toshiaki Manabe, MD, $\mathrm{PhD}{ }^{2}$ \\ Hiromi Wada, MD, PhD, ${ }^{1}$ and Fumihiro Tanaka, MD, $\mathrm{PhD}^{1}$
}

${ }^{1}$ Department of Thoracic Surgery, Faculty of Medicine, Kyoto University, Kyoto, Japan

${ }^{2}$ Laboratory of Anatomic Pathology, Kyoto University Hospital, Kyoto, Japan ${ }^{3}$ Center for Anatomical Studies, Kyoto University Graduate School of Medicine, Kyoto, Japan

\begin{abstract}
Purpose: Aurora-A, also known as STK15/BTAK, is a member of the protein serine/ threonine kinase family, and experimental studies have revealed that Aurora-A plays critical roles in cell mitosis and in carcinogenesis. However, no clinical studies on Aurora-A expression in non-small-cell lung cancer (NSCLC) have been reported. Thus, the present study was conducted to assess the clinical significance of Aurora-A status.

Experimental Design: A total of 189 consecutive patients with resected pathologic (p-)stage I-IIIA, NSCLC were retrospectively reviewed, and immunohistochemical staining was used to detect Aurora-A expression.

Results: Aurora-A expression was negative in 31 patients (16.4\%); among Aurora-A positive patients, 124 patients showed pure diffuse cytoplasmic Aurora-A expression and the other 34 patients showed perimembrane Aurora-A expression. Perimembrane Aurora-A tumors showed the highest proliferative index (PI) (mean PIs for negative, diffuse cytoplasmic, and perimembrane tumors: 49.2, 41.7, and 63.5, respectively; $P<.001$ ). Five-year survival rates of Aurora-A negative, diffuse cytoplasmic, and perimembrane patients were $67.8 \%, 66.7 \%$, and $47.6 \%$, respectively, showing the poorest postoperative survival in perimembrane patients $(P=.033)$. Subset analyses revealed that perimembrane Aurora-A expression was a significant factor to predict a poor prognosis in squamous cell carcinoma patients, not in adenocarcinoma patients. A multivariate analysis confirmed that perimembrane Aurora-A expression was an independent and significant factor to predict a poor prognosis.

Conclusions: Perimembrane Aurora-A status was a significant factor to predict a poor prognosis in correlation with enhanced proliferative activity in NSCLC.
\end{abstract}

Key Words: Aurora-A-Lung cancer-Proliferation-Surgery-Prognosis.

Primary lung cancer is the leading cause of cancer deaths in most industrialized countries, and non-

Received October 17, 2005; accepted July 7, 2006; published online: November 28, 2007.

Address correspondence and reprint requests to: Fumihiro Tanaka, MD, PhD; E-mail: ftanaka@kuhp.kyoto-u.ac.jp

Published by Springer Science+Business Media, LLC @ 2008 The Society of Surgical Oncology, Inc. small-cell lung cancer (NSCLC) accounts for 75-80\% of primary lung cancer. Surgery is the most effective therapeutic modality for the cure, but the postoperative prognosis remains poor. ${ }^{1-3}$ To improve the prognosis, establishment of biological markers other than tumor-node-metastasis (TNM) factors that determine prognosis and response to a particular treatment is essential. Although many possible biological markers such as mutations of $p 53$ and $k$-ras 
genes have been reported, none of them has been established as a marker in decision-making of the treatment of NSCLC. In addition, most NSCLC patients present with advanced stages and are appropriate candidates for surgery. For inoperable patients, chemotherapy with or without radiotherapy may be given. Recently, tyrosine kinase inhibitors targeting epidermal growth factor receptor (EGFR), gefitinib and erlotinib, have been introduced into the therapy of NSCLC, and experimental and clinical studies have revealed that these agents induce dramatic antitumor effects for tumors with activating mutations within the EGFR kinase domain. ${ }^{4,5}$ Thus, other molecular abnormalities in NSCLC should be revealed in the development of useful prognostic markers and in the development of effective molecular targeting agents.

Aurora-A, also known as BTAK, STK15, Aurora2, ARKI, or AIKI, is a member of the serine/threonine kinase family. As Aurora-A is involved in proper centrosome functions and chromosome segregation during normal cell mitosis, ${ }^{6-8}$ its abnormalities such as overexpression as well as gene amplification may contribute to development and progression of malignant tumors. ${ }^{9-11}$ In clinical studies, Aurora-A abnormalities have been reported in a variety of malignant tumor including glioma, ${ }^{12}$ medullobrastoma, ${ }^{13}$ gastric cancer, ${ }^{14}$ esophageal cancer, ${ }^{15,16}$ pancreatic cancer, ${ }^{17}$ ovarian cancer, ${ }^{18}$ breast cancer, ${ }^{19,20}$ bladder cancer, ${ }^{21}$ and hepatocellular carcinoma. ${ }^{22}$ In all these clinical studies, Aurora-A gene was amplified or Aurora-A expression was upregulated in tumor tissues compared with normal tissues, which also support experimental results suggesting that AuroraA abnormalities are involved in carcinogenesis. In addition, many studies showed that Aurora-A abnormalities were positively correlated with aggressive tumor behavior such as poorly differentiated tumor grade, invasion, and nodal metastasis, ${ }^{15,16,19,20-22}$ but some studies showed no correlation ${ }^{12,17}$ or an inverse correlation. ${ }^{18}$ Some studies also assessed a prognostic significance of Aurora-A status; most studies showed that Aurora-A abnormalities were correlated with a significant poor prognosis, ${ }^{13,16,21,22}$ but one study failed to show. ${ }^{20}$ These results may suggest that clinical significant of Aurora-A status in malignant tumors remains controversial. Moreover, no clinical study on Aurora-A status in NSCLC has been reported. Thus, we conducted a clinical study on Aurora-A expression in resected NSCLC and assessed its clinical significance in correlation with other biomarkers including proliferative activity.

\section{PATIENTS AND METHODS}

\section{Patients}

We retrospectively reviewed a total of 191 consecutive patients with pathologic (p)-stage I-IIIA NSCLC who underwent complete tumor resection and nodal dissection without any preoperative therapy at the Department of Thoracic Surgery, Kyoto University, between January 1, 1985 and December 31, 1990. The p-stage was reevaluated and determined according to the current tumornode-metastasis (TNM) classification as revised in 1997. Histological type was also redetermined according to the classification by the World Health Organization (WHO) as revised in 2004. Two patients who experienced operation-related death were excluded from the study. Thus, a total of 189 patients were finally evaluated in the present study. For all these patients, the inpatient medical records, chest x-ray films, whole-body computed tomography (CT) films, bone and gallium scanning data, and records of surgery were reviewed without knowledge of the results of immunohistochemical staining (IHS) or the terminal deoxynucleotidyl transferasemediated dUTP-biotin nick end-labeling (TUNEL) staining.

Details of postoperative adjuvant therapy were described in previous studies. ${ }^{23,24}$ In brief, cisplatinbased chemotherapy, radiation, and oral administration of tegafur (a fluorouracil derivative drug) were prescribed postoperatively for 47,30 , and 49 patients, respectively. Intraoperative therapy was not performed on any patient.

The day of thoracotomy was considered the starting day for counting postoperative survival days. The median and mean follow-up periods were 1697 and 1983 days, respectively (range, 506381 days). This study has been approved by the Ethics Committee of Faculty of Medicine, Kyoto University.

\section{Tissue Preparation}

All tumor specimens were fixed immediately in $10 \mathrm{vol} \%$ formalin, and then embedded in paraffin. Serial $4-\mu \mathrm{m}$ sections were prepared from each sample and used for routine hematoxylin and eosin (HE) staining, the TUNEL staining to detect apoptotic cells, and IHS to determine Aurora-A expression status, p53 status, intratumoral microvessel density (IMVD), and proliferative index (PI). 


\section{Immunohistochemistry}

Aurora-A expression was evaluated using a standard streptavidin-biotinylated horseradish peroxidase detection system. Dewaxed sections were incubated overnight with a rabbit polyclonal antibody (KR051, Transgenic Co. Ltd., Kumamoto, Japan; it had been produced and its reliability had been confirmed by western blotting on a commercial basis) diluted at 1:100. Diaminobenzidine-tetrahydrochloride $(0.03 \%)$ containing $0.1 \%$ hydrogen peroxide was used as a chromogen, and sections were counterstained with hematoxylin. For negative control slides, rabbit immunoglobulin $G$ was used instead of the primary antibody. Colon cancer sections that are known to express Aurora-A were used as positive control slides.

Aurora-A expression on tumor cells was first classified into negative or positive expression; a slide was judged to be Aurora-A positive expression, regardless of the staining intensity. For slides with positive Aurora-A expression, the staining pattern was, then, classified as follows: diffuse cytoplasmic staining (Fig. 1B), perimembrane staining (Fig. 1C). Slides were evaluated by two pathologists (E.O. and Y.T.) independently, without knowledge of any patient characteristic.

PI, IMVD, and p53 status were also determined with IHS as described previously. ${ }^{23,25}$ Briefly, an antiPCNA monoclonal antibody (PC-10, mouse immunogloblin G [IgG] 2a, kappa; DAKO Japan, Kyoto, Japan) diluted at 1:50 was used as the primary antibody to determine PI $;{ }^{23}$ PI was defined as the percentage of PCNA-positive staining tumor cells. IMVD was determined with an anti-CD34 monoclonal antibody QBEnd10 (mouse IgG1, kappa; DAKO Japan) diluted at 1:50; the 10 most vascular areas within a section were selected for evaluation of angiogenesis, and the average counts of CD34-positive vessels were recorded as IMVD in each case. ${ }^{25}$ An antihuman p53 monoclonal antibody DO-7 (mouse IgG2b, kappa, $250 \mu \mathrm{g} / \mathrm{mL}$, DAKO Japan) was used to determine p53 status. When the percentage of positive-staining cells exceeded $5 \%$, the slide was judged to exhibit aberrant expression of p53. ${ }^{23}$

\section{Detection of Apoptosis}

The TUNEL staining was performed using the In Situ Death Detection Kit, POD (Boehringer Manheim, Manheim, Germany) following the manufacture's protocol as described previously. ${ }^{23}$ The specificity of the TUNEL staining of apoptotic cells
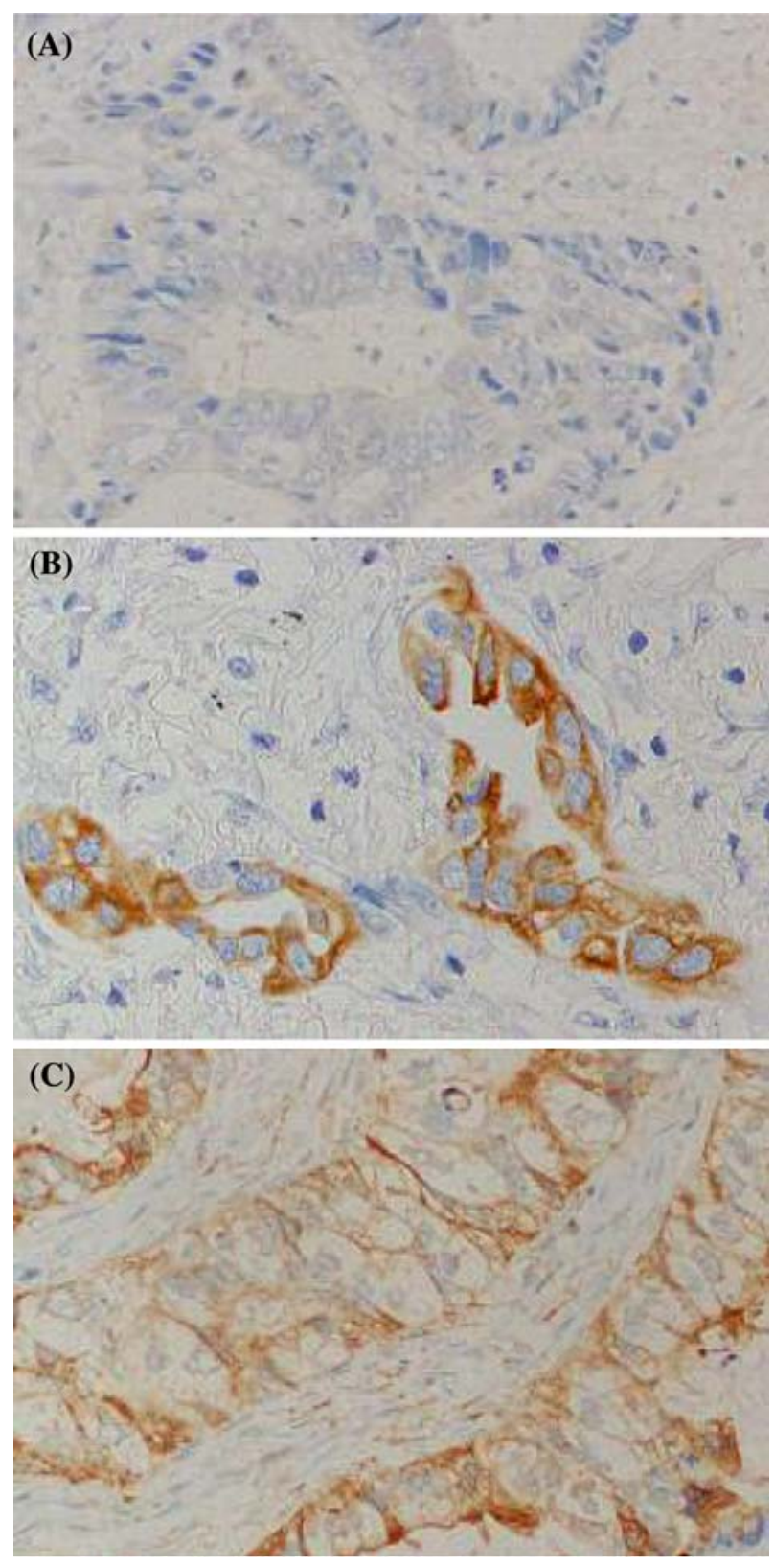

FIG. 1. Aurora-A expression in non-small-cell carcinoma (NSCLC) as detected with imuunohistochemical staining. A Negative staining. B diffuse cytoplasmic staining. C Peri-membrane staining.

was confirmed by making the negative and the positive control slides at every staining. As negative control slides, sections incubated with the TUNEL reaction mixture without TdT were used. Sections treated with DNase I (Stratagene, La Jolla, CA) before the TUNEL reaction were used as positive control slides. Apoptotic cells were determined with careful observation of TUNEL-staining sections and serial HE-staining sections, and TUNEL-positive 
staining cells, if they represented the histological features of necrosis in HE-staining sections, were not considered to be apoptotic cells. In each case, a total of 10,000 tumor cells, consisting of 1000 tumor cells each in 10 different fields, were evaluated at high magnification $(400 \times)$. The apoptotic index (AI) was defined as the number of apoptotic cells per 1000 tumor cells.

\section{Statistical Analysis}

Counts were compared by the chi-square test. Continuous data were compared using the $t$ test if the distribution of the samples was normal, or using the Mann-Whitney $U$ test if the sample distribution was asymmetrical. The postoperative survival rate was analyzed by the Kaplan-Meier method, and differences in the survival rates were assessed by the logrank test. Death from any cause was included in calculation of postoperative survival. Multivariate analysis of the prognostic factors was performed using Cox's proportional hazard model. Differences were considered significant when the $P$ value was less than .05. All statistical manipulations were performed using the SPSS ver.10 for the Windows software system (SPSS Inc., Chicago, IL).

\section{RESULTS}

\section{Expression of Aurora-A in NSCLC}

Expression of Aurora-A was not observed in normal adjacent lung tissues in any patient. Expression of Aurora-A in tumor cells was negative in 31 patients $(16.4 \%)$ and positive in the other158 patients. Among Aurora-A positive patients, 124 patients showed diffuse cytoplasmic staining, 6 patients showed pure perimembrane staining, and 28 patients showed both diffuse cytoplasmic staining and perimembrane staining (mixed staining). Because the number of patients showing pure perimembrane staining was very small $(n=6)$, both patients with pure perimembrane staining and patients with mixed staining were classified into perimembrane patients. Thus, there were 31 negative patients (16.4\%), 124 diffuse cytoplasmic patients $(65.6 \%)$, and 34 perimembrane patients (18.0\%) (Table 1).

The incidence of perimembrane staining was significantly higher in higher p-stages; perimembrane staining was seen in only $11.5 \%$ (12 of 104) of p-stage I patients, but seen in as high as $25.8 \%$ (22 of 85) of p-stage II-IIIA patients (Table 1). There was no significant correlation between Aurora-A expression status and any other patients' characteristic (Table 1).

The mean PI for perimembrane Aurora-A expression patients high tumor was significantly higher than that for Aurora-A negative or that for diffuse cytoplasmic Aurora-A expression patients (PIs: 63.5, 49.2, and 41.7, respectively; $P<.001$ ) (Table 2). There was no significant difference in the mean IMVD or in the mean AI according to the status of Aurora-A expression; there was no significant correlation between Aurora-A expression status and p53 status (Table 2).

\section{Expression of Aurora-A and Postoperative Survival}

For all 189 patients, 5-year survival rates of Aurora-A negative patients, diffuse cytoplasmic AuroraA patients, and perimembrane Aurora-A patients were $67.8 \%, 66.7 \%$, and $47.6 \%$, respectively; perimembrane Aurora-A patients showed the poorest postoperative survival $(P=.033)$ (Table 3 and Fig. 2).

Subset analyses revealed that perimembrane Aurora-A expression was a significant factor to predict a poor prognosis in squamous cell carcinoma patients, not in adenocarcinoma patients $(P=.007$ and $P=.894$, respectively) (Table 3 ).

A multivariate analysis of prognosis factors using a Cox proportional hazard model confirmed that perimembrane Aurora-A expression was an independent and significant factor to predict a poor prognosis in NSCLC (Table 4).

\section{DISCUSSION}

The present study is the first clinical study on Aurora-A expression in NSCLC and has revealed the clinical significance as follows: (1) positive Aurora-A expression is exclusively observed in tumor cells, not in normal cells; (2) there are two Aurora-A expression patterns, diffuse cytoplasmic staining and perimembrane staining; (3) perimembrane staining is significantly correlated to higher p-stage and higher proliferative activity; and (4) perimembrane staining is a significant and independent factor to predict a poor prognosis, especially in squamous cell carcinoma patients. As mentioned in the introduction section, previous experimental and clinical studies have demonstrated that Aurora-A is overexpressed and upregulated in tumor cells and/or tissues, ${ }^{8-20,22,26}$ which is also true in NSCLC as demonstrated in the present study. The centrosomes play important roles 
TABLE 1. Characteristics of patients and Aurora expression

\begin{tabular}{|c|c|c|c|c|c|}
\hline & \multirow[b]{3}{*}{ No. of patients $(\%)$} & \multicolumn{3}{|c|}{ MAGE-D4 expression } & \multirow[b]{3}{*}{$P$ value } \\
\hline & & \multirow[b]{2}{*}{ Negative } & \multicolumn{2}{|l|}{ Positive } & \\
\hline & & & Diffuse cytoplasmic & Perimembrane & \\
\hline All patients & $189(100)$ & $31(16.4 \%)$ & $124(65.6 \%)$ & $34(18.0 \%)$ & \\
\hline Age $($ mean \pm SD) $62.4 \pm 9.6$ & $60.5 \pm 10.2$ & $62.3 \pm 9.2$ & $64.3 \pm 9.1$ & 0.256 & \\
\hline Sex & & & & & .137 \\
\hline Male & $133(70.4)$ & $26(19.5 \%)$ & $86(64.7 \%)$ & $21(15.8 \%)$ & \\
\hline Female & $56(29.6)$ & $5(8.9 \%)$ & $38(67.9 \%)$ & $13(23.2 \%)$ & \\
\hline Performance status (PS) & & & & & .353 \\
\hline 0 & $164(86.8)$ & $28(17.1 \%)$ & $109(66.5 \%)$ & $27(16.5 \%)$ & \\
\hline $1-2$ & $25(13.2)$ & $3(12.0 \%)$ & $15(60.0 \%)$ & $7(28.0 \%)$ & \\
\hline Histologic type & & & & & .302 (Sq vs Ad) \\
\hline Squamous cell $(\mathrm{Sq})$ & 64 (33.9) & $8(12.5 \%)$ & $41(64.1 \%)$ & $15(23.4 \%)$ & \\
\hline Adeno (Ad) & $108(57.1)$ & $19(17.6 \%)$ & $73(67.6 \%)$ & $16(14.8 \%)$ & \\
\hline Large cell (La) & $10(5.3)$ & $4(40.0 \%)$ & $4(40.0 \%)$ & $2(20.0 \%)$ & \\
\hline Others & $7(3.7)$ & $0(0.0 \%)$ & $5(85.7 \%)$ & $1(14.3 \%)$ & \\
\hline Tumor differentiation ${ }^{*}$ & & & & & .393 \\
\hline Well & $68(37.4)$ & $9(13.2 \%)$ & $49(72.1 \%)$ & $10(14.7 \%)$ & \\
\hline Moderately & $67(36.8)$ & $13(19.4 \%)$ & $43(64.2 \%)$ & $11(16.4 \%)$ & \\
\hline Poorly & $47(25.8)$ & $9(19.1 \%)$ & $26(55.3 \%)$ & $12(25.5 \%)$ & \\
\hline Pathologic (p-) stage & & & & & .007 (for all p-stage) \\
\hline I & $104(55.0)$ & $12(11.5 \%)$ & $80(76.9 \%)$ & $12(11.5 \%)$ & \\
\hline II & 22 (11.6) & $4(18.2 \%)$ & $11(50.0 \%)$ & $7(31.8 \%)$ & \\
\hline IIIA & $63(33.3)$ & $15(23.8 \%)$ & $33(52.4 \%)$ & $15(23.8 \%)$ & \\
\hline
\end{tabular}

* Other histologic types were excluded in the analyses.

TABLE 2. Correlation between Aurora expression and other biomarkers

\begin{tabular}{|c|c|c|c|c|}
\hline & \multicolumn{3}{|c|}{ Aurora expression } & \multirow[b]{3}{*}{$P$ value } \\
\hline & \multirow[b]{2}{*}{ Negative } & \multicolumn{2}{|l|}{ Positive } & \\
\hline & & Diffuse cytoplasmic & Perimembrane & \\
\hline Intratumoral microvessel density (IMVD) ${ }^{*}$ & $191.2 \pm 16.3$ & $174.7 \pm 8.9$ & $167.6 \pm 14.6$ & .584 \\
\hline Proliferative index $(\mathrm{PI})(\%)$ & $49.2 \pm 4.8$ & $41.7 \pm 2.5$ & $63.5 \pm 3.8$ & $<.001$ \\
\hline Apoptotic index (AI) (/1000 tumor cells) & $13.5 \pm 3.2$ & $20.5 \pm 2.0$ & $15.9 \pm 2.5$ & .154 \\
\hline p53-status: Aberrant expression $(+)$ & $12 / 31(38.7 \%)$ & $55 / 124(44.4 \%)$ & $15 / 34(44.1 \%)$ & .848 \\
\hline
\end{tabular}

Values were shown as the mean \pm standard error (SE).

* Evaluated with an anti-CD34 antibody.

TABLE 3. Aurora expression and postoperative survival in non-small-cell lung cancer (NSCLC) patients

\begin{tabular}{|c|c|c|c|c|}
\hline & \multicolumn{3}{|c|}{ 5-year survival rate $(\%)$} & \multirow[b]{4}{*}{$P$ value } \\
\hline & \multicolumn{3}{|c|}{ AURORA expression } & \\
\hline & \multirow[b]{2}{*}{ Negative } & \multicolumn{2}{|l|}{ Positive } & \\
\hline & & Diffuse cytoplasmic & Perimembrane & \\
\hline All patients & $67.8 \%$ & $66.7 \%$ & $47.6 \%$ & .033 \\
\hline \multicolumn{5}{|c|}{ Subset analysis, histologic type } \\
\hline Squamous cell $(\mathrm{Sq})$ & $75.0 \%$ & $74.2 \%$ & $34.5 \%$ & .007 \\
\hline \multirow{2}{*}{\multicolumn{5}{|c|}{ Pathologic (p-)stage }} \\
\hline & & & & \\
\hline I & $91.7 \%$ & $72.9 \%$ & $58.3 \%$ & .098 \\
\hline II & $75.0 \%$ & $68.6 \%$ & $57.1 \%$ & .579 \\
\hline IIIA & $50.4 \%$ & $50.9 \%$ & $28.9 \%$ & .088 \\
\hline
\end{tabular}

in the maintenance of genomic instability by establishing bipolar spindles during mitosis and by ensuring equal segregation of replicated chromosome into two daughter cells. ${ }^{27}$ Aurora-A is involved in normal centrosome functions, and its abnormalities such as gene amplification and overexpression may lead to 
TABLE 4 Multivariate analysis of prognostic factors (Cox's proportional hazard model)

\begin{tabular}{lccc}
\hline Factors & Beta & $P$ value & Hazard ratio (95\% confidence interval) \\
\hline Gender (male/female) & -0.539 & .096 & $0.583(0.309-1.101)$ \\
Age & 0.006 & .714 & $1.006(0.977-1.035)$ \\
Performance status (0/1/2) & 0.183 & .571 & $1.201(0.637-2.264)$ \\
Histologic type (Nonadenocarcinoma/adenocarcinoma) & -0.050 & .162 & $0.951(0.887-1.020)$ \\
Pathologic stage (I/II/IIIa) & 0.551 & .001 & $1.734(1.311-2.295)$ \\
Perimembrane Aurora expression (No/Yes) & -0.362 & .032 & $0.343(0.169-0.699)$ \\
\hline
\end{tabular}

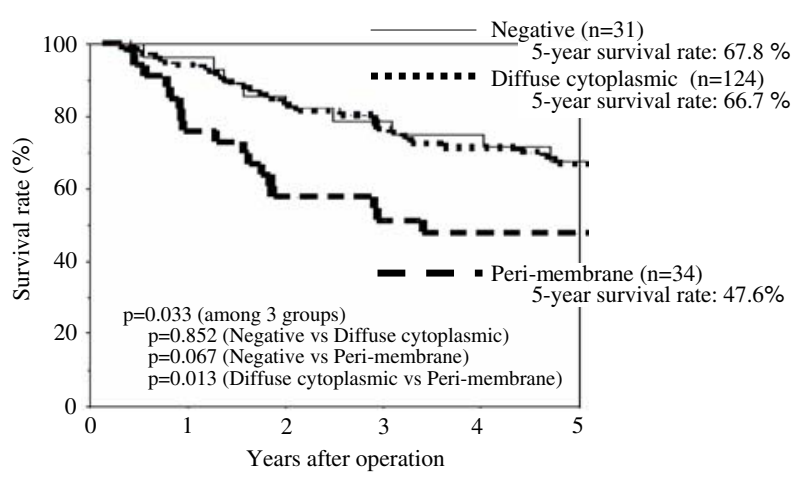

FIG. 2. Survival curves of patients who underwent complete resection for pathologic (p-) stage I-IIIA non-small-cell lung cancer. Comparison according to Aurora-A expression status.

centrosome function disorder, chromosomal instability, and carcinogenesis. ${ }^{26}$ Aurora- $A$ gene amplification usually contributes to overexpression of Aurora-A gene and Aurora-A protein, but also common is overexpression without gene amplification. ${ }^{22}$ These results may suggest that Aurora-A expression is regulated not only by gene amplification but also by other mechanisms. ${ }^{22}$ In the present study, only Aurora-A protein expression was assessed immunohistochemically, and comparative study of overexpression and amplification should be conducted in future.

In previous clinical studies on Aurora-A expression assessed with IHS, Aurora-A expression was classified simply into negative or positive, and its clinical significance was examined. ${ }^{13,15-18,20}$ Some studies showed a significantly positive correlation between Aurora-A overexpression and aggressive tumor behavior such as poor differentiation and nodal metastasis, ${ }^{15,16,20}$ but others showed no ${ }^{17}$ or an inverse correlation. ${ }^{18}$ In addition, among three studies where prognostic significance of Aurora-A overexpression was assessed, two studies showed a significantly poor prognosis in patients with Aurora-A overexpression $^{13,16}$ and one failed to show. ${ }^{20}$ These conflicting results on clinical significance of Aurora-A expression status might be partly due to the evaluation system. In fact, there was no difference in the proliferative activity as represented as PI or in the prognosis between negative Aurora-A tumors and diffuse cytoplasmic Aurora-A tumors in the present study (Tables 2 and 3); when perimembrane AuroraA expression was separated and analyzed, tumors with perimembrane Aurora-A expression showed an aggressive tumor phenotype correlated with a higher PI and a poor prognosis. The biochemical implication of perimembrane Aurora-A expression is unknown, staining patterns should be taken into consideration in evaluation of Aurora-A expression status in clinical samples.

Another reason for conflicting results of clinical significance of Aurora-A expression status might be difference in the histologic type. Two clinical studies on esophageal squamous cell carcinoma showed that Aurora-A overexpression was significantly correlated with aggressive tumor behavior. ${ }^{15,16}$ In contrast, two clinical studies on adenocarcinoma, one on pancreatic cancer ${ }^{17}$ and one on breast cancer, ${ }^{20}$ failed to show a significant correlation between Aurora-A status and tumor differentiation or prognosis. In fact, a subset analysis according to histologic types in the present study showed that perimembrane Aurora-A expression was a significant factor to predict a poor prognosis in lung squamous cell carcinoma, but not in lung adenocarcinoma (Table 3). These results may suggest that clinical impact of Aurora-A expression status is enhanced in squamous cell carcinoma and reduced in adenocarcinoma. Further clinical studies on a variety of malignant tumors will give some answers to the issue.

We analyzed correlations between Aurora-A status and other biomarkers such as proliferative activity and p53 status. There has been no clinical study where Aurora-A status is assessed in correlation with proliferative activity. Considering experimental results that Aurora-A is a centrosome-associated kinase that contributes to regulation of cell mitosis, ${ }^{8,26}$ Aurora-A expression status may be correlated with proliferative activity in clinical samples as shown in the present study (Table 2). However, it remains unknown why a specific Aurora-A expression pat- 
tern, the perimembrane expression, is correlated with higher proliferative activity. Experimental studies have also suggested an interaction between Aurora-A and $\mathrm{p} 53,{ }^{28-30}$ suggesting that the $\mathrm{p} 53$ inhibit oncogenic activity of Aurora- $\mathrm{A}^{28}$ and that Aurora-A, especially when overexpressed, inactivate tumorsuppressor activity of p53. ${ }^{29,30}$ One clinical study also documented that Aurora-A overexpression was significantly correlated with p53 mutation in hepatocellular carcinoma. ${ }^{22}$ These results strongly suggest that Aurora-A in concert with p53 contributes to the development and progression of malignant tumors. In the present study, we failed to show a significant correlation between Aurora-A expression status and p53 status as assessed with IHS. In a future study, $p 53$ gene mutation status in correlation with Aurora-A expression should be examined.

More recently, increasing studies on Aurora-A gene polymorphisms and risk of malignant tumors have been reported. ${ }^{31-36}$ Two common single nucleotide polymorphisms in the coding regions are $91 \mathrm{~T}->\mathrm{A}$ transversion and $169 \mathrm{G}->\mathrm{A}$ transversion, which result in phenylalanine/isoleucine substitution at amino acid 31 (F31I) and valine/isoleucine substitution at amino acid 57 (V57I), respectively. Several studies have shown such polymorphisms are associated with increased risk of occurrence of a variety of malignant tumors including esophageal, ${ }^{31,35}$ ovarian, ${ }^{32}$ and breast $^{33,34}$ cancers. In future, correlation between Aurora-A polymorphism and risk of development of primary lung cancer should be investigated. In conclusion, Aurora-A expression status was a significant prognostic factor in correlation with proliferative activity in NSCLC. To confirm the clinical significance, future prospective studies should be conducted.

\section{ACKNOWLEDGMENT}

We thank Miss Seiko Sakai for helpful preparation of the manuscript.

\section{OPEN ACCESS}

This article is distributed under the terms of the Creative Commons Attribution Noncommercial License which permits any noncommercial use, distribution, and reproduction in any medium, provided the original author(s) and source are credited.

\section{REFERENCES}

1. Mountain CF. Revisions in the international system for lung cancer. Chest 1997; 111:1710-7.
2. Naruke T, Goya T, Tsuchiya R, Suemasu K. Prognosis and survival in resected lung carcinoma based on the new international staging system. J Thorac Cardiovasc Surg 1998; 96:440-7.

3. Tanaka F, Yanagihara K, Otake Y, et al. Surgery for nonsmall cell lung cancer: postoperative survival based on the revised tumor-node-metastasis classification and its time trend. Eur J Cardiothorac Surg 2000; 18:147-55.

4. Lynch TJ, Bell DW, Sordella R, et al. Activating mutations in the epidermal growth factor receptor underlying responsiveness of non-small-cell lung cancer to gefitinib. $N$ Engl $J$ Med 2004; 350:2129-39.

5. Paez JG, Janne PA, Lee JC, et al. EGFR mutations in lung cancer: correlation with clinical response to gefitinib therapy. Science 2004; 304:1497-500.

6. Goover DM, Leibowitz MH, McLean DA, Parry H. Mutations in aurora prevent centrosome separation leading to the formation of monopolar spindles. Cell 1995; 81:95-105.

7. Bischoff JR, Anderson L, Zhu Y, et al. A homologue of Drosophila aurora kinase is oncogenic and amplified in human colorectal cancers. EMBO J 1998; 17:3052-65.

8. Zhou H, Kuang J, Zhong L, et al. Tumor amplified kinase STK15/BTAK induces centrosome amplification, aneuploidy and transformation. Nat Genet 1998; 20:189-93.

9. Goepfert TM, Adigun YE, Zhong L, Gay J, Medina D, Brinkley WR. Centrosome amplification and overexpression of Aurora A are early events in rat mammary carcinogenesis. Cancer Res 2002; 62:4115-22.

10. Ke YW, Dou Z, Zhang J, Yao XB. Function and regulation of Aurora/Ip11p kinase family in cell division. Cell Res. 2003; 13:69-81.

11. Balanos-Garcia VM. Aurora kinases. Int J Biochem Cell Biol 2005; 37:1572-7.

12. Klein A, Reichardt W, Jung V, Zang KD, Meese E, Urbschat S. Overexpression and amplification of STK15 in human gliomas. Int J Oncol 2004; 25:1789-94.

13. Neben K, Korshunov A, Benner A, et al. Microarray-based screening for molecular markers in medulloblastoma revealed STK 15 as independent predictor for survival. Cancer Res 2004; 64:3103-11.

14. Sakakura C, Hagiwara A, Yasuoka R, et al. Tumour-amplified kinase BTAK is amplified and overexpressed in gastric cancers with possible involvement in aneuploid formation. $\mathrm{Br}$ J Cancer 2001; 84:824-31.

15. Tong T, Zhong Y, Kong J, et al. Overexpression of Aurora-A contributes to malignant development of human esophageal squamous cell carcinoma. Clin Cancer Res 2004; 10:7304-10.

16. Tanaka E, Hashimoto $Y$, Ito $T$, et al. The clinical significance of Aurora-A/STK15/BTAK expression in human esophageal squamous cell carcinoma. Clin Cancer Res 2005; 11:1827-34.

17. Li D, Zhu J, Firozi PF, et al. Overexpression of oncogenic STK15/BTAK/Aurora A kinase in human pancreatic cancer. Clin Cancer Res 2003; 9:991-7.

18. Gritsko TM, Coppola D, Paciga JE, Yang L, Sun M, Shelley SA, Fiorica JV, Nicosia SV, Cheng JQ. Activation and over expression of centrosome kinase BTAK/Aurora-A in human ovarian cancer. Clin Cancer Res 2003; 9:1420-6.

19. Miyoshi Y, Iwao K, Egawa C, Noguchi S. Association of centrosomal kinase STK15/BTAK mRNA expression with chromosomal instability in human breast cancers. Int $J$ Cancer 2001; 92:370-3.

20. Royce ME, Xia W, Sahin AA, et al. STK15/Aurora-A expression in primary breast tumors is correlated with nuclear grade but not with prognosis. Cancer 2004; 100:12-9.

21. Sen S, Zhou H, Zhang RD, et al. Amplification/overexpression of a mitotic kinase gene in human bladder cancer. $J$ Natl Cancer Inst 2002; 94:1320-9.

22. Jeng YM, Peng SY, Lin CY, Hsu HC. Over expression and amplification of Aurora-A in hepatocellular carcinoma. Clin Cancer Res 2004; 10:2065-71. 
23. Tanaka F, Kawano Y, Li M, et al. Prognostic significance of apoptotic index in completely resected non-small-cell lung cancer. J Clin Oncol 1999; 17:2728-36.

24. Tanaka F, Yanagihara K, Ohtake Y, et al. p53 status predicts the efficacy of postoperative oral administration of tegafur for completely resected non-small cell lung cancer. Jpn J Cancer Res (Cancer Sci) 1999; 90:432-8.

25. Tanaka F, Otake Y, Yanagihara K, et al. Evaluation of angiogenesis in non-small cell lung cancer : comparison between anti-CD34 antibody and anti-CD105 antibody. Clin Cancer Res 2001; 7:3410-5.

26. Bischoff JR, Anderson L, Zhu Y, et al. A homologue of Drosophila aurora kinase is oncogenic and amplified in human colorectal cancers. EMBO J 1998; 17:3052-65.

27. Zimmerman W, Sparks CA, Doxsey SJ. Amorphous no longer: the centrosome comes into focus. Curr Opin Cell Biol 1999; $11: 122-8$.

28. Chen SS, Chang PC, Cheng YW, Tang FM, Lin YS. Suppression of the STK15 oncogenic activity requires a transactivation-independent p53 function. EMBO J 2002; 21:4491-9.

29. Liu Q, Kaneko S, Yang L, Feldman RI, Nicosia SV, Chen J, Cheng JQ. Aurora-A abrogation of p53 DNA binding and transactivation activity by phosphorylation of serine $215 . J$ Biol Chem 2004; 279:52175-82.
30. Katayama H, Sasai K, Kawai H, et al. Phosphorylation by aurora kinase A induces Mdm2-mediated destabilization and inhibition of p53. Nat Genet 2004; 36:55-62.

31. Miao X, Sun T, Wang Y, Zhang X, Tan W, Lin D. Functional STK15 Phe31Ile polymorphism is associated with the occurrence and advanced disease status of esophageal squamous cell carcinoma. Cancer Res 2004; 64:2680-3.

32. Dicioccio RA, Song H, Waterfall C, et al. STK15 polymorphisms and association with risk of invasive ovarian cancer. Cancer Epidemiol Biomarkers Prev 2004; 13:1589-94.

33. Egan KM, Newcomb PA, Ambrosone CB, et al. STK15 polymorphism and breast cancer risk in a population-based study. Carcinogenesis 2004; 25:2149-53.

34. Sun T, Miao X, Wang J, Tan W, Zhou Y, Yu C, Lin D. Functional Phe31Ile polymorphism in Aurora A and risk of breast carcinoma. Carcinogenesis 2004; 25:2225-30.

35. Kimura MT, Mori T, Conroy J, Nowak NJ, Satomi S, Tamai $\mathrm{K}$, Nagase $\mathrm{H}$. Two functional coding single nucleotide polymorphisms in STK15 (Aurora-A) coordinately increase esophageal cancer risk. Cancer Res 2005; 65:3548-54.

36. Ewart-Toland A, Dai Q, Gao YT, et al. Aurora-A/STK15 $\mathrm{T}+91 \mathrm{~A}$ is a general low penetrance cancer susceptibility gene: a meta-analysis of multiple cancer types. Carcinogenesis 2005; 6:1368-73. 